\title{
The Therapeutic Options in Childhood Epilepsy: From Pharmaceuticals to Nutraceuticals
}

\section{Samuel N Uwaezuoke*}

Department of Paediatrics, University of Nigeria Teaching Hospital (UNTH) Ituku-Ozalla, Enugu, Nigeria

*Corresponding author: Samuel N Uwaezuoke, Department of Paediatrics, University of Nigeria Teaching Hospital (UNTH) Ituku-Ozalla, Enugu, Nigeria, Tel: +2348033248108; E-mail: snuwaezuoke@yahoo.com

Received date: November 19, 2015; Accepted date: December 23, 2015; Published date: December 30, 2015

Copyright: (C2015 Uwaezuoke SN. This is an open-access article distributed under the terms of the Creative Commons Attribution License, which permits unrestricted use, distribution, and reproduction in any medium, provided the original author and source are credited.

\begin{abstract}
Epilepsy is one of the most common neurological disorders of childhood. Treatment of epileptic seizures is governed by weighing the benefit- risk of using anti-epileptic drugs. An important limitation of their use is that, in at least one third of patients, seizures are resistant to the available older and new-generation anti-epileptic drugs. This challenge makes the non-pharmacologic mode of treatment an attractive option. With the global demonstration of the therapeutic benefits of the ketogenic diet signaling a possible paradigm shift, evaluating its risk profile will help to establish its benefit-risk ratio. Multifaceted side effects of the diet have been reported in several studies but have not precluded its use. To promote its world-wide acceptability, its components should be modified to include locallyavailable food stuffs. The present review aims to highlight and compare the benefit-risk ratio of using anti-epileptic drugs and the ketogenic diet in the treatment of refractory childhood epilepsy.
\end{abstract}

Keywords: Childhood epilepsy; Anti-epileptic drugs; Refractory seizures; Ketogenic diets; Paradigm shift

\section{Introduction}

Epilepsy is one of the most common neurological disorders of childhood [1,2]. By definition, it is a condition in which a subject is predisposed to recurrent seizures due to a disorder of the central nervous system: a seizure referring to a sudden, involuntary, timelimited alteration in behavior, including a change in motor activity, in autonomic function, in consciousness, or in sensation, accompanied by an abnormal electrical discharge in the brain [3]. Determination of the underlying epileptic syndrome is crucial because of its far-reaching implications for pharmacologic treatment and prognosis [4]. An epileptic syndrome may consist of more than one seizure type. Thus, in the classification system of the epilepsies by the International League against Epilepsy (ILAE), the primary distinction is based on the patient's seizure type- either partial or generalized [5]. Generally, commencement of anti-epileptic drugs should be preceded by a number of considerations such as assessment of the risk of further seizures, and establishing if the seizures are non-epileptic so that treatment can be targeted at the underlying cause [6]. Furthermore, treatment of epileptic seizures is governed by weighing the risk of treatment against the benefit of using anti-epileptic drugs. The goal of treatment with anti-epileptic drugs is to prevent the recurrence of seizures while avoiding the side effects from these drugs. Accurate identification of the seizure type is a prerequisite for choosing the appropriate drug for the best outcome.

Since the historical evolution of pharmaceuticals in the treatment of epileptic seizures which started with the use of bromide, several antiepileptic drugs have emerged till date. Most of these drugs have distinctive side-effects which may be fatal on rare occasions; thus their successful use considers both their efficacy and side effects. After initiation of treatment with anti-epileptic drugs, the treatment should be monitored to evaluate their effectiveness and to determine their safety profile [7]. Another major challenge in the use of anti-epileptic drugs is the issue of non-compliance which has been well documented in a study [8]. Drug interactions are also a major problem. Seizures may remain intractable despite treatment with anti-epileptic drugs, while prolonged periods of treatment partly contribute to noncompliance with these pharmaceuticals.

Since the mid-1990s, there has been renewed scientific interest in the ketogenic diet as therapeutic option for drug-resistant childhood epilepsy. The diet has been widely and successfully used to treat children with epilepsy since the 1920s until the advent of anti-epileptic drugs in the 1930s. In 2008, the first randomized controlled trial of the ketogenic diet demonstrated that $38 \%$ of children on the diet had a $>50 \%$ reduction in seizures compared with only $6 \%$ in the control group [9]. Despite its efficacy in treating drug-resistant epilepsy, shortterm and long-term side effects have been observed [10]. Interestingly, the use of nutraceuticals has equally become attractive in other noncommunicable diseases [11]. Nutraceuticals refer to foods or food components which provide medical benefits including prevention and/or treatment of disease [11], and the ketogenic diet appears to be a fitting eponym [12]. The present review aims to highlight and compare the benefit-risk ratio of using anti-epileptic drugs and the ketogenic diet in the treatment of refractory childhood epilepsy.

\section{Anti-Epileptic Drugs: Weighing the Benefit-Risk Ratio}

Evaluating the benefit to risk ratio in children with epilepsy is extremely important in determining the need for treatment, the choice of drugs and the use of monitoring tools such as laboratory tests and other investigations [13]. Active epilepsy per se is associated with significant risks in terms of increased mortality, susceptibility to neuropsychiatric morbidity and physical injury, and reduced quality of life traceable to restricted lifestyle, stigma and prejudice. By preventing the occurrence of seizures, anti-epileptic drugs reduce or eliminate altogether seizure-related risks; but other risks may arise due to the side effects of the drugs, all of which have a relatively narrow 
therapeutic index [13]. As a rule, there are no major differences in the degree of efficacy between anti-epileptic drugs effective in any given seizure type. A comparison of the safety profiles of anti-epileptic drugs however shows that different medications have different side-effect profiles [7], and these represent a major factor in determining the choice of treatment [13]. In as many as $60-70 \%$ of newly treated patients, current anti-epileptic drugs result in satisfactory seizure control and a favorable benefit-risk balance for the great majority of them despite considerable differences in response; unfortunately, in $20-30 \%$ of patients, epilepsy remains refractory [14] .

Furthermore, assessment of benefit to risk ratio should also consider patient-related factors such as the type and severity of the epilepsy, age, gender, childbearing potential, medical and drug history, associated disease, use of concomitant medication and the expected patient's compliance [13]. For instance, in some benign epilepsy syndromes, the risk of side effects from anti-epileptic drugs may outweigh potential benefits in terms of seizure control; thus, treatment is generally not indicated. On the other hand, the serious morbidity and mortality associated with severe epileptic encephalopathies may justify aggressive treatment even with drugs associated with a relatively high risk of life-threatening side effects [13].

In one study that compared four drugs, all of them had similar efficacy but were associated with distinctive and varying side effects [15]. Several systemic and neurotoxic effects occurred with the four drugs (primidone, phenobarbital, phenytoin and carbamazepine) but some were significantly more common or less common with specific drugs [15]. Typically, phenobarbital was associated with the lowest incidence of motor disturbance (ataxia, uncoordination, nystagmus, and tremor) early in treatment, while phenytoin resulted in more dysmorphic and idiosyncratic side effects (gum hypertrophy, hirsuitism, acne and rash) than the other drugs. These four antiepileptic drugs were among the early set of pharmaceuticals introduced for the treatment of epilepsy since the debut of bromide in 1857. From the mid-70s till the late-90s, other anti-epileptic drugs like clonazepam, valproate, clorazepate, lamotrigine, fosphenytoin and topiramate emerged and were marketed in the United States [16]. In addition, much newer anti-epileptic drugs are also available and include gabapentin, felbamate, vigabatrin, tiagabine, oxcarbazepine, and zonisamide. Three of the new drugs namely felbamate, gabapentin and lamotrigine have long been made available for prescription [17]. A comprehensive list of the side-effects of both the older and newer antiepileptic drugs is further outlined alongside with their interactions with other anti-epileptic drugs and medications as follows [16,17]:

\section{Older antiepileptic drugs}

Carbamazepine -Skin: urticaria, Stevens-Johnson syndrome, exfoliative dermatitis, petechiae, etc. Neurologic: drowsiness, dizziness, diplopia, ataxia, confusion, headache, blurred vision, nystagmus, tinnitus, etc. Gastrointestinal: abdominal pain, diarrhea, constipation, anorexia, glossitis, stomatitis, etc. Cardiovascular: arrhythmias, hypotension, syncope, edema, etc. Genitourinary: urinary frequency, acute urinary retention, etc. Carbamazepine can increase the levels of phenytoin and phenobarbital and decrease the levels of clonazepam, ethosuximide, primidone, sodium valproate, phenytoin and phenobarbital. On its concomitant interactions with other medications, the level of carbamazepine can be increased by erythromycin, isoniazid, and cimetidine.

Phenytoin-skin eruptions, peripheral neuropathy, gingival hypertrophy, hypertrichosis and hirsuitism. Phenytoin may increase or decrease the levels of carbamazepine, phenobarbital, primidone and sodium valproate. Its level can be increased by drugs like chloramphenicol, cimetidine, isoniazid, sulfonamides, trimethoprim, and chlorpheniramine

Sodium valproate-Gastrointestinal: nausea, vomiting, anorexia, heartburn, diarrhea, abdominal cramps, constipation, and increased appetite. Neurologic: hand tremor, sedation, drowsiness, aggressiveness, hyperactivity, ataxia and headache. Skin: alopecia, rash, epistaxis. The drug may increase the level of phenobarbital. Its level may be increased by salicylates

Phenobarbital-drowsiness, blunting of memory and motor performance, depression, excitability, skin eruptions. The drug decreases the level of clonazepam but may decrease or increase the levels of phenytoin and carbamazepine. Its level may be increased by chloramphenicol.

Primidone- sedation. Its interaction with other antiepileptic drugs is similar to phenobarbital but its level is increased by isoniazid

\section{Newer antiepileptic drugs}

Gabapentin- fatigue, dizziness, nystagmus, decreased alertness, tremor, sleep disturbances, hypotension, dry mouth, nausea, diarrhea and rash. A characteristic feature of this drug is its non-interaction with other antiepileptic drugs and other medications.

Lamotrigine-diplopia, drowsiness, ataxia, headache, skin rashes including Stevens-Johnson syndrome. The drug does not affect the levels of other antiepileptic drugs but its half-life can be prolonged or shortened by phenytoin and carbamazepine respectively.

Felbamate-insomnia, nausea, decreased appetite, dizziness, fatigue, ataxia and lethargy. The levels of phenytoin and valproate increase with the drug. However, it decreases the level of carbamazepine while its level is decreased by the concomitant use of other antiepileptic drugs, especially those that induce hepatic enzymes.

Topiramate-somnolence, dizziness, ataxia, speech disorders, and paresthesias. The drug does not affect the levels of other drugs but its concentration can be decreased by phenytoin and carbamazepine.

Notably, each of the anti-epileptic drugs may be effective for some types of seizures but may be ineffective for other types. In addition, the risk profile of each drug may make its use more beneficial or less beneficial in children. For example, carbamazepine is effective alone or with other anti-epileptic drugs in the treatment of partial seizures, especially complex partial seizures, in generalized tonic-clonic seizures, and in combinations of these seizure types [16]. However, the drug is ineffective for absence, myoclonic, and atonic seizures. Although it has been found to worsen some generalized seizures with spike-and-wave EEG patterns in children, it is increasingly preferred to phenobarbital in pediatric patients because it appears to have less untoward effect than phenobarbital on cognition and behavior [16]. Nevertheless, carbamazepine has several side effects ranging from dermatologic, hematologic, gastrointestinal, neurologic, cardiovascular, and hepatic to metabolic reactions; some of these reactions are potentially fatal albeit rare. Despite these challenges, the benefit-risk ratio still favors its use in the treatment of childhood epilepsy. Some of the newer antiepileptic drugs were limited in their use with respect to the age of the patients. For instance, gabapentin and lamotrigine were initially used in only adult patients while felbamate was approved in pediatric patients [17]. However, more recently the NICE (National Institute for health and Care Excellence) guidelines for antiepileptic drugs 
recommend the following as first-line and alternative first-line medications in the indicated seizure types [18]:

For the treatment of focal seizures in children, young people and adults, the first- line drugs are carbamazepine and lamotrigine. The alternative first line drugs include levetiracetam, oxcarbazepine, sodium valproate while the adjunctive treatment (if first line treatment is not effective or not tolerated) is carbamazepine, clobazam, gabapentin, lamotrigine, levetiracetam, oxcarbazepine, sodium valproate, and topiramate.

For the treatment of generalized tonic clonic seizures in children, young people and adults, the recommended first- line drugs are sodium valproate, and lamotrigine (if sodium valproate is not suitable). The alternative first- line drugs include carbamazepine, and oxcarbazepine. The adjunctive treatment (if first line treatment is not effective or not tolerated) are clobazam, lamotrigine, levetiracetam, sodium valproate, topiramate

For the treatment of absence seizures in children, young people and adults, the first-line medications are ethosuximide and sodium valproate (offer first if additional tonic clonic seizures are likely). The alternative first line treatment is lamotrigine. Adjunctive treatment (if first line treatment is not effective or not tolerated) consists of a combination of ethosuximide, lamotrigine or sodium valproate.

For the treatment of myoclonic seizures in children, young people and adults, the first-line drug is sodium valproate. The alternative firstline drugs include levetiracetam, and topiramate. The adjunctive treatment (if first line treatment is not effective or not tolerated) comprises levetiracetam, sodium valproate, and topiramate.

For the treatment of tonic and atonic seizures in children, young people and adults, the first-line drug is sodium valproate. The adjunctive treatment (if first line treatment is not effective or tolerated) is lamotrigine.

Obviously, an important limitation of the use of the current pharmaceuticals is that, in at least one third of patients, seizures are refractory to the available anti-epileptic drugs [19-21]. The phenomenon of drug resistance has not been significantly affected by the emergence of second-generation anti-epileptic drugs, as no more than $10-15 \%$ of patients with severe refractory epilepsy become seizure free by treatment with these agents $[22,23]$. Thus, in a major review on the current trends in anti-epileptic drug therapy, one author suggests the following strategies to overcome these limitations: an increasing reliance on therapeutic guidelines and evidence-based medicine; a more rational patient-tailored selection of anti-epileptic drugs and drug dosages; a better definition of the indications of new antiepileptic drugs; novel approaches to anti-epileptic drug combinations; and the development of newer anti-epileptic drugs, novel modes of administration, and novel therapeutic targets [24]. These strategies have however not completely eliminated the issue of drug resistance in childhood epilepsy. This makes the non-pharmacologic mode of treatment an attractive option especially with the renaissance of ketogenic diets.

\section{Ketogenic Diets in Refractory Epilepsy: Weighing the Benefit-Risk Ratio}

A ketogenic diet is a strict diet comprising minimal carbohydrate and protein intake and increased fat intake: usually $85 \%$ full fat, $10 \%$ protein (sufficient for growth) and 5\% carbohydrate with total energy intake restricted to $75 \%$ of recommended daily intake and fluid intake restricted to $80 \%$ recommended daily intake [25]. The ratio of grams of fats to grams of protein and carbohydrates is basically $4: 1$. Heavy cream, butter, and oil are some of the foods that can become staples of children on a ketogenic diet: a diet which requires vitamin and mineral supplementation. The ketogenic diet is used primarily as a nonpharmacologic therapy for the control of refractory childhood epilepsy [26]. The treatment is typically recommended when conventional anti-epileptic drugs have failed or anti-epileptic drugs therapy results in unacceptable side effects. About $30 \%$ of children with epilepsy will have either of these outcomes [27]. In a published consensus report, the International Ketogenic Diet Study Group recommends that ketogenic diets should be strongly considered in a child who failed to respond to two or three anticonvulsant therapies, particularly in those patients with symptomatic generalized epilepsies [28].

The ketogenic diet mimics the body's response to starvation by the utilization of fat as the primary source of energy in the absence of an adequate dietary carbohydrate source [29]. Under normal metabolism, the body metabolizes carbohydrates into glucose which is the fastest source of energy for the body and typically the sole energy source for the brain. In a fasting state, amino acids cannot provide an adequate energy source for the brain and fatty acids cannot cross the bloodbrain barrier. The liver uses the fatty acids to produce ketone bodies, which can cross the blood-brain barrier and substitute for glucose as an energy source. The mechanism of how ketosis controls seizures remains unclear; however, one theory suggests that ketones have an anticonvulsant effect when crossing the blood-brain barrier [29]. Ketogenic diets are also being increasingly studied for neurological disorders other than epilepsy, such as Alzheimer's disease, autism and brain tumor; insights from basic science research have helped to clarify the mechanisms by which metabolism-based therapy may be helpful, in terms of both an anticonvulsant and possibly a neuroprotective effect [30]. Although the mechanisms for the latter effect are not yet well elucidated, it is likely that the neuroprotection results from enhanced neuronal energy reserves, which improve the ability of neurons to resist metabolic challenges, and possibly through antioxidant and anti-inflammatory effects [31].

There are multiple variations of ketogenic diets, but the most commonly prescribed are the classic ketogenic diet, the modified Atkins diet, the low-glycemic index treatment diet, the medium-chain triglyceride diet, and the modified medium-chain triglyceride diet [32]. The efficacy of the diets has been demonstrated mostly in children with generalized seizures and those between 1 and 10 years of age [33]. Other recent systematic reviews [34,35] and a meta-analytical study [36] have also evaluated and reported about the success and benefits of the ketogenic diet as a therapeutic option for childhood epilepsy: a finding which has been documented in many retrospective and prospective studies [9,37-43]. For instance in one prospective study [40], the change in frequency of seizures in 51 children with intractable seizures treated with the ketogenic diet was investigated. These children (aged 1 to 8 years, with more than 10 seizures per week, whose electroencephalogram showed generalized epileptiform abnormalities or multifocal spikes, and who had failed to respond to least 2 appropriate anti-epileptic drugs) were hospitalized, fasted, and placed on a 4:1 ketogenic diet for up to 1 year. After 6 months, 28 (55\%) had at least a $50 \%$ decrease from baseline while at 1 year, $40 \%$ of those starting the diet had a greater than $50 \%$ decrease in seizures. Five patients $(10 \%)$ were free of seizures at 1 year (Table 1$)$. The side effects attributed to the diet included lethargy, severe dehydration or acidosis, 
behavioral changes, susceptibility to infections, severe constipation, and vomiting [40].

\begin{tabular}{|l|l|l|l|}
\hline Initiated Diet & 3 Months & $\mathbf{6}$ Months & 12 Months \\
\hline Number on diet & $45(88)$ & $35(69)$ & $24(47)$ \\
\hline$>90 \%$ control of seizures $\dagger$ & $13(25)$ & $15(29)$ & $11(22)$ \\
\hline $50 \%-90 \%$ control of seizures & $15(29)$ & $12(24)$ & $9(18)$ \\
\hline$<50 \%$ control of seizures & $17(33)$ & $8(16)$ & $4(8)$ \\
\hline Discontinued diet & $6(12)$ & $14(27)$ & $23(45)$ \\
\hline Lost to follow-up & 0 & 2 & 4 \\
\hline
\end{tabular}

Table 1: Outcome of the ketogenic $\operatorname{diet}^{*}\left[{ }^{*} \mathrm{~N}=51\right.$ : Values are number (percentage), $\dagger$ Numbers free of seizures were the following: 6 (3 months), 6 (6 months), and 5 (12 months)].

Similar findings were documented in the first randomized controlled trial to test the efficacy of the diet in drug-resistant epilepsy [9]. The researchers enrolled 145 children who were aged between 2 and 16 years; had at least daily seizures (or more than seven seizures per week); had failed to respond to at least two antiepileptic drugs; and had not been treated previously with the ketogenic diet. Seventy three children were assigned to the ketogenic diet group and 72 children to the control group. After 3 months, the mean percentage of baseline seizures was significantly lower in the diet group than in the controls (62.0\% vs $136.9 \%$, $75 \%$ decrease, $95 \%$ CI $42.4-107.4 \%$; $<<0.0001)$. Twenty eight children (38\%) in the diet group had greater than $50 \%$ seizure reduction compared with four $(6 \%)$ controls $(\mathrm{p}<0.0001)$, and five children $(7 \%)$ in the diet group had greater than $90 \%$ seizure reduction compared with no controls $(\mathrm{p}=0.0582)$. The most frequent side-effects reported at 3-month review were constipation, vomiting, lack of energy, and hunger [9]. Furthermore, other studies have reported various degrees of improvement in seizure control among pediatric patients with refractory epilepsy. In one retrospective study [43], seizure control improved in $67 \%$ of the patients while in another prospective study [42], the workers documented that the mean seizure frequency decreased from 25 per day to less than 2 per day after one year of the diet. In another retrospective study of 32 infants treated with the diet, its overall effectiveness was noted to be similar to that reported for older children [38]. For instance, $19.4 \%$ of these infants became seizure-free, and an additional $35.5 \%$ had $>50 \%$ reduction in seizure frequency; the diet was particularly effective for patients with infantile spasms/myoclonic seizures, and was generally well-tolerated with 96.4\% maintaining appropriate growth parameters [38]. Improvements in behavior and function, alertness, activity level, and socialization were also reported. However, side effects occurred in one patient each and included renal stone, gastritis, ulcerative colitis, alteration of mentation, and hyperlipidemia.

With the global demonstration of the therapeutic benefits of ketogenic diets and the possible paradigm shift from anti-epileptic drugs, evaluating the risk profile of the diet is very important in establishing its benefit-risk ratio. Despite its proven benefits, multifaceted side effects have been reported in several studies, and in summary consist of gastrointestinal disturbances (diarrhea, vomiting, nausea, constipation, gastro-esophageal reflux) [44-46], nephrolithiasis [47,48], poor growth in children [46,49-52], increased bruising [53], osteopenia/osteoporosis [51,54], sepsis and bacterial overgrowth $[41,45,54]$, pneumonia $[44,45,47,54]$, acute pancreatitis $[45,54,55]$, hypoglycemia [41], impaired mood [41], cardiomyopathy $[41,44,54,56]$, as well as hypercholesterolemia/hypertriglyceridemia $[41,44,54]$.

Apart from these side effects, patient compliance is another major challenge; it mainly depends on the types of ketogenic diet and the age group of the patients [28]. Specifically, children who are fed by the enteral route usually demonstrate very high compliance rates, whereas a diet with a fat: non-fat ratio of more than 4.5: 1 usually leads to poor compliance [28]. Older children and adolescents usually show difficulty adhering to the strict diet ratio of $4: 1$. Thus, a lower fat: nonfat ratio is often used in this population [28]. Nonetheless, the risk profile of and patient poor compliance to the strict ketogenic diet have not precluded its acceptability. The diet appears to be culturally acceptable to the caregivers once modified to include food items from their environmental setting. Recently, the Keilah Foundation (a nongovernmental organization) has successfully used the ketogenic diet to manage children with epilepsy in South Africa; the diet has also been successfully implemented in Uganda where locally-available and familiar food stuffs were utilized [57]. The typical ketogenic diet that is viable to follow in Uganda is based on the local food products namely beans with cabbage and mukene (a fish caught in Lake Victoria). The recipe consists of $14 \mathrm{~g}$ red kidney beans, $25 \mathrm{~g}$ butter, $9 \mathrm{~g}$ dried mukene, $10 \mathrm{~g}$ oil, $14 \mathrm{~g}$ cabbage and $14 \mathrm{~g}$ cream [57]. Interestingly, this finding may be the propelling force to drive the acceptability of the ketogenic diet beyond the Western world to the developing world, and to promote nutraceuticals as the new paradigm in the treatment of refractory childhood epilepsy.

\section{Conclusion}

Following the rebirth of the use of the ketogenic diet as a therapeutic option in drug-resistant childhood epilepsy, pediatric neurologists now consider it as possible first-line therapy in infantile spasms and status epilepticus. Although the diet is often prescribed alongside one or more anti-epileptic drugs, its efficacy as a sole therapeutic agent has been well documented in several studies. Like anti-epileptic drugs, several short-term and long-term side effects have also been reported; but these treatment challenges have not made the ketogenic diet a less attractive option. Nevertheless, the diet should still be used as an adjunctive therapy with the first-line or second-line antiepileptic drugs in the management of refractory seizures. To promote its global acceptability, the components of the diet should be modified to include locally-available food stuffs; this local adaptation has been implemented in Uganda, eastern Africa.

\section{References}

1. Shinnar S, Pellock JM (2002) Update on the epidemiology and prognosis of pediatric epilepsy. J Child Neurol 17: S4-17.

2. Hirtz D, Thurman DJ, Gwinn-Hardy K, Mohamed M, Chaudhuri AR, et al. (2007) How common are the "common" neurologic disorders? Neurology 68: 326-337.

3. Gumnit RJ, Leppik IE (1991) The epilepsies. In: Rosenberg R, ed. Comprehensive Neurology. New York, Raven Press 311-336.

4. Leppik IE (1990) Epileptic syndromes: Genetic, diagnostic, and therapeutic aspects: introductory remarks and symposium overview. Epilepsia 31: S1-S2.

5. (1989) Commission on Classification and Terminology of the International League against Epilepsy: proposal for the classification of epilepsy and epileptic syndromes. Epilepsia 30: 389-399. 
6. Leppik IE (1997) When to start and when to stop treatment for seizures In: Leppik IE, ed. Contemporary diagnosis and management of the patient with epilepsy. Handbooks in Health Care, Pennsylvania 49-56.

7. Leppik IE (1997) Principles of treatment. In: Leppik IE, ed. Contemporary diagnosis and management of the patient with epilepsy. Handbooks in Health Care, Pennsylvania 57-66.

8. Leppik IE (1988) Compliance during treatment of epilepsy. Epilepsia 29: S79-84.

9. Neal EG, Chaffe H, Schwartz RH, Lawson MS, Edwards N, et al. (2008) The ketogenic diet for the treatment of childhood epilepsy: a randomized controlled trial. Lancet Neurol 7: 500-506.

10. Hartman AL, Vining EP (2007) Clinical aspects of the ketogenic diet. Epilepsia 48: 31-42.

11. Scicchitano P, Cameli M, Maiello M, Modesti PA, Muiesan ML, et al. (2014) Nutraceuticals and dyslipidemia: Beyond the common therapeutics. J Funct Foods 6: 11-32.

12. Scrivens SD (2014) Eponyms in medical nutrition and nutraceuticals: the ketogenic diet for seizure control. J Med Nutr Nutraceut 3: 76-78.

13. Perucca E, Beghi E, Dulac O, Shorvon S, Tomson T (2000) Assessing risk to benefit ratio in antiepileptic drug therapy. Epilepsy Res 41: 107-139.

14. Schmidt D (2009) Drug treatment of epilepsy: options and limitations. Epilepsy Behav 15: 56-65.

15. Mattson RH, Cramer JA, Collins JF, Smith DB, Delgado-Escueta AV, et al. (1985) Comparison of carbamazepine, phenobarbital, phenytoin, and primidone in partial and secondarily generalized tonic-clonic seizures. $\mathrm{N}$ Engl J Med 313: 145-151.

16. Leppik IE (1997) Standard antiepileptic drugs (AEDs). In: Leppik IE, ed. Contemporary diagnosis and management of the patient with epilepsy. Handbooks in Health Care, Pennsylvania 67-94.

17. Leppik IE (1997) Newly approved antiepileptic drugs. In: Leppik IE, ed. Contemporary diagnosis and management of the patient with epilepsy. Handbooks in Health Care, Pennsylvania 95-103.

18. (2012) The epilepsies: the diagnosis and management of the epilepsies in adults and children in primary and secondary care. NICE clinical guideline 137, NICE.

19. Snead OC III, Hosey LC (1985) Exacerbation of seizures in children by carbamazepine. N Engl J Med 313: 916-921.

20. Perucca E (1998) Pharmacoresistance in epilepsy. How should it be defined? CNS Drugs 10: 171-179.

21. Kwan P, Brodie MJ (2000) Early identification of refractory epilepsy. N Engl J Med 342: 314-319.

22. Perucca E (2001) The clinical pharmacology and therapeutic use of the new antiepileptic drugs. Fundam Clin Pharmacol 15: 405-417.

23. Walker MC, Sander JWAS (1996) The impact of the new antiepileptic drugs on the prognosis of epilepsy. Seizure freedom should be the ultimate goal. Neurology 46: 912-914.

24. Perucca E (2003) Current trends in anti-epileptic drug therapy. Epilepsia 44: S41-S47.

25. Freeman JM (2007) What is the Ketogenic Diet?, The Ketogenic Diet: A Treatment for Children and Others with Epilepsy. (4thedn) Demos Medical Publishing, New York.

26. Hartman AL, Vining EP (2007) Clinical aspects of the ketogenic diet. Epilepsia 48: 31-42.

27. Kwan P, Brodie MJ (2001) Effectiveness of first antiepileptic drug. Epilepsia 42: 1255-1260.

28. Kossoff EH, Zupec-Kania BA, Amark PE, Ballaban-Gil KR, Bergqvist AGC et al. (2009) Optimal clinical management of children receiving the ketogenic diet: recommendations of the International Ketogenic Diet Study Group. Epilepsia 50: 304-317.

29. Runyon AM, So T (2012) The use of ketogenic diet in pediatric patients with epilepsy. ISRN Pediatrics.

30. Kossoff EH, Hartman AL (2012) Ketogenic diets: new advances for metabolism-based therapies. Curr Opin Neurol 25:173-178.
31. Gasior M, Rogawski MA, Hartman AL (2006) Neuroprotective and disease-modifying effects of the ketogenic diet. Behav Pharmacol 17: 431-439.

32. Hobdell EF, Tonyes L (2007) Diets for epilepsy. Touch Briefings: US Pediatric Review 2: 45-46.

33. Henderson CB, Filloux FM, Alder SC, Lyon JL, Caplin DA (2006) Efficacy of the ketogenic diet as a treatment option for epilepsy: meta-analysis. J Child Neurol 21: 193-198.

34. Lefevre F, Aronson N (2000) Ketogenic diet for the treatment of refractory epilepsy in children: A systematic review of efficacy. Pediatrics 105: E46.

35. Keene DL (2006) A systematic review of the use of the ketogenic diet in childhood epilepsy. Pediatr Neurol 35: 1-5.

36. Li HF, Zou Y, Ding G (2013) Therapeutic success of the ketogenic diet as a treatment option for epilepsy: a meta-analysis. Iran J Pediatr 23: 613-620.

37. Kang HC, Lee YM, Kim HD, Lee JS, Slama A (2007) Safe and effective use of the ketogenic diet in children with epilepsy and mitochondrial respiratory chain complex defects. Epilepsia 48: 82-88.

38. Nordli DR, Kuroda MM, Carroll J, Koenigsberger DY, Hirsch LJ, et al. (2001) Experience with the ketogenic diet in infants. Pediatrics 108: 129-133.

39. Hassan AM, Keene DL, Whiting SE, Jacob PJ, Champagne JR, et al (1999) Ketogenic diet in the treatment of refractory epilepsy in childhood. Pediatr Neurol 21: 548-552.

40. Vining EPG, Freeman JM, Ballaban-Gil K, Camfield CS, Camfield PR et al., (1998) The Ketogenic Diet Multi-Center Study Group. A multicenter study of the efficacy of the ketogenic diet. Arch Neurol 55: 1433-1437.

41. Coppola G, Veggiotti P, Cusmai R, Bertoli S, Cardinali S et al. (2002) The ketogenic diet in children, adolescents and young adults with refractory epilepsy: an Italian multicentric experience. Epilepsy Res 48: 221-227.

42. Pulsifer MB, Gordon JM, Brandt J, Vining EP, Freeman JM (2001) Effects of ketogenic diet on development and behavior: preliminary report of a prospective study. Dev Med Child Neurol. 43: 301-306.

43. Kinsman SL, Vining EP, Quaskey SA, Mellits D, Freeman JM (1992) Efficacy of the ketogenic diet for intractable seizure disorders: review of 58 cases. Epilepsia 33: 1132-1136.

44. Nam SH, Lee BL, Lee CG, Yu HJ, Joo EY, et al. (2011) The role of ketogenic diet in the treatment of refractory status epilepticus. Epilepsia 2: e181-e184.

45. Kang HC, Kim YJ, Kim DW, Kim HD (2005) Efficacy and Safety of the Ketogenic Diet for Intractable Childhood Epilepsy: Korean Multicentric Experience. Epilepsia 46: 272-279.

46. Groesbeck DK, Bluml RM, Kossoff EH (2006) Long-term use of the ketogenic diet in the treatment of epilepsy. Dev Med Child Neurol 48: 978-981.

47. Suo C, Liao J, Lu X, Fang K, Hu Y et al (2013) Efficiency and safety of the ketogenic diet in Chinese children. Seizure 22: 174-178.

48. Sampath A, Kossoff EH, Furth SL, Pyzik PL, Vining EPG (2007) Kidney Stones and the Ketogenic Diet: Risk Factors and Prevention. J Child Neurol 22: 375-378.

49. Williams S, Basualdo-Hammond C, Curtis R, Schuller R (2002) Growth retardation in children with epilepsy on ketogenic diet. J Am Diet Assoc 102: 405-407.

50. Vining EPG, Pyzik P, McGrogan J, Hladky H, Anand A, et al. (2007) Growth of children on the ketogenic diet. Dev Med Child Neurol 44: 796-802.

51. Bergqvist AGC, Schall JI, Stallings VA, Zemel BS (2008) Progressive bone mineral content loss in children with intractable epilepsy treated with ketogenic diet. Am J Clin Nutr 88: 1678-1684.

52. Kim JT, Kang H, Song J, Lee MJ, Lee YJ, et al. (2013) Catch-up growth after long-term implementation and weaning from ketogenic diet in pediatric epileptic patients. Clin Nutr 32:98-103.

53. Berry-Kravis E, Booth G, Taylor A, Valentino LA (2001) Bruising and the ketogenic diet: evidence for diet-induced changes in platelet function. Ann Neurol 49: 98-103. 
Citation: Uwaezuoke SN (2015) The Therapeutic Options in Childhood Epilepsy: From Pharmaceuticals to Nutraceuticals . J Neurol Neurophysiol 6: 344. doi:10.4172/2155-9562.1000344

Page 6 of 6

54. Kang HC, Chung DE, Kim DW, Kim HD (2004) Early- and late-onset complications of the ketogenic diet for intractable epilepsy. Epilepsia 45 1116-1123.

55. Stewart WA, Gordon K, Camfield P (2001) Acute pancreatitis causing death in a child on the ketogenic diet. J Child Neurol 16: 682.
56. Bank TM, Shemie SD, Rosenblatt B, Bernard C, Mackie AS (2008) Sudden cardiac death in association with the ketogenic diet. Pediatr Neurol 39: 429-431.

57. Megaw K, Wilmshurst JM (2015) The Keilah Foundation: making the ketogenic diet viable for children in Africa. Epilepsia 56: 514-516. 